\title{
Solvability of Dirichlet Problem For a Fractional Partial Differential equation by using energy inequality and Faedo-Galerkin method
}

Research Article

\author{
Zainouba Chebana $^{1 *}$, Taki-Eddine Oussaeif ${ }^{1 \dagger}$, Adel Ouannas $^{1,3 \ddagger}$ Iqbal M. Batiha $^{2,3 \S}$ \\ 1 Department of Mathematics and Informatics, Oum El Bouaghi University, Oum El Bouaghi, Algeria \\ 2 Department of Mathematics, Faculty of Science and Technology, Irbid National University, 2600 Irbid, Jordan \\ 3 Nonlinear Dynamics Research Center (NDRC), Ajman University, Ajman, UAE
}

\begin{abstract}
In this paper, we establish sufficient conditions for the existence and uniqueness of the solution for a class of initial-boundary value problems with Dirichlet condition for a class of fractional partial differential equations. The results are established by a method based on a priori estimate "energy inequality" and the Faedo-Galerkin method.

MSC: $\quad 35 \mathrm{~A} 05,35 \mathrm{~A} 07,35 \mathrm{~K} 50,35 \mathrm{Q} 80$

Keywords: Fractional partial differential equation • Energy inequality • Faedo-Galerkin method • Existence and uniqueness
\end{abstract}

\section{Introduction}

Differential equations have a remarkable ability to predict the world around us. They are used in a wide variety of disciplines, from biology, economics, physics, chemistry and engineering. They can describe exponential growth and decay, the population growth of species or the change in investment return over time. As an example, consider the propagation of light and sound in the atmosphere, and of waves on the surface of a pond. All of them may be described by the same second-order partial differential equation, the wave equation, which allows us to think of light and sound as forms of waves, much like familiar waves in the water. Conduction of heat, the theory of

\footnotetext{
* E-mail: chebanazainouba@yahoo.com

$\dagger$ E-mail: taki_maths@live.fr

¥ E-mail:dr.ouannas@gmail.com

§E-mail: ibatiha@inu.edu.jo
} 
which was developed by Joseph Fourier, is governed by another second-order partial differential equation, the heat equation. It turns out that many diffusion processes, while seemingly different, are described by the same equation; the Black-Scholes equation in finance is, for instance, related to the heat equation. Also, for example in medicine for modelling cancer growth or the spread of disease, in engineering for describing the movement of electricity, in chemistry for modelling chemical reactions and to computer radioactive half life, in economics to find optimum investment strategies, in physics to describe the motion of waves, pendulums or chaotic systems. It is also used in physics with Newton's Second Law of Motion and the Law of Cooling and in Hooke's Law for modeling the motion of a spring or in representing models for population growth and money flow circulation.

Fractional calculus has gained importance during the past three decades due to its applicability in diverse fields of science and engineering. The notions of fractional calculus may be traced back to the works of Euler, but the idea of fractional difference is very recent. Fractional differential equations (FDEs) are obtained by generalizing differential equations to an arbitrary order. Since fractional differential equations are used to model complex phenomena, they play a crucial role in engineering, physics and applied mathematics. Therefore they have been generating increasing interest from engineers and scientist in recent years. Since FDEs have memory, nonlocal relations in space and time, complex phenomena can be modeled by using these equations. Due to this fact, materials with memory and hereditary effects, through strongly anomalous media. Indeed, we can find numerous applications in viscoelasticity, electro-chemistry, signal processing, control theory, porous media, fluid flow, rheology, diffusive transport, electrical networks, electromagnetic theory and probability, signal processing, and many other physical processes are diverse applications of FDEs [1-6].

Recently, there has been a significant development in fractional differential and partial differential equations (see, e.g., [7] and the references therein).

The study of existence and uniqueness, periodicity, asymptotic behavior, stability, and methods of analytic and numerical solutions of fractional differential equations have been studied extensively in a large cycle works; Especially, The study of existence and uniqueness of solution of fractional partial differential equations are then proved by the well-known Lax-Milgram theorem, Energy estimate and fixed point theorem. Among them, we only mention here the papers [8-21].

The present paper is devoted to the study of initial-boundary value problem for a parabolic equation with timefractional derivative with Dirichlet condition by Faedo-Galerkin method and a priori estimate, which has not been studied so far.

\section{Preliminaries and functional spaces}

Let $\boldsymbol{\Gamma}(\cdot)$ denote the gamma function. For any positive integer $0<\alpha<1$, Caputo derivative and Riemann Liouville derivative are, respectively, defined as follows: 
(i) The left Caputo derivatives:

$$
{ }_{0}^{C} D_{t}^{\alpha} u(x, t):=\frac{1}{\Gamma(1-\alpha)} \int_{0}^{t} \frac{\partial u(x, \tau)}{\partial \tau} \frac{1}{(t-\tau)^{\alpha}} d \tau
$$

the right Caputo derivatives:

$$
{ }_{t}^{C} D_{T}^{\alpha} u(x, t):=\frac{-1}{\Gamma(1-\alpha)} \int_{t}^{T} \frac{\partial u(x, \tau)}{\partial \tau} \frac{1}{(\tau-t)^{\alpha}} d \tau .
$$

(ii) The left Riemann-Liouville derivatives:

$$
{ }_{0}^{R} D_{t}^{\alpha} u(x, t):=\frac{1}{\Gamma(1-\alpha)} \frac{\partial}{\partial t} \int_{0}^{t} \frac{u(x, \tau)}{(t-\tau)^{\alpha}} d \tau
$$

the right Riemann-Liouville derivatives:

$$
{ }_{t}^{R} D_{T}^{\alpha} v(t)=\frac{-1}{\Gamma(1-\alpha)} \frac{\partial}{\partial t} \int_{t}^{T} \frac{u(x, \tau)}{(t-\tau)^{\alpha}} d \tau .
$$

Many authors think that the Caputo's version is more natural because it allows the handling of inhomogeneous initial conditions in a easier way. Then definitions (2.1) and (2.2) are linked by the following relationship, which can be verified by a direct calculation:

$$
{ }_{0}^{R} D_{t}^{\alpha} u(x, t)={ }_{0}^{C} D_{t}^{\alpha} u(x, t)+\frac{u(x, 0)}{\Gamma(1-\alpha) t^{\alpha}} .
$$

Definition 2.1.

[10] For any real $\sigma>0$, we define the semi-norm:

$$
|u|_{l_{H}(I)}^{2}:=\left\|{ }_{0}^{R} D_{t}^{\sigma} u\right\|_{L_{2}(I)}^{2}
$$

and norm:

$$
\|u\|_{l_{H^{\sigma}(\Omega)}}:=\left(\|u\|_{L_{2}(I)}^{2}+|u|_{l_{H_{0}^{\sigma}(I)}}^{2}\right)^{\frac{1}{2}},
$$

we then define ${ }^{l} H_{0}^{\sigma}(I)$ as the closure of $C_{0}^{\infty}(I)$ with respect to the norm $\|\cdot\|_{l_{H_{0}^{\sigma}(I)}}$.

\section{Definition 2.2.}

For any real $\sigma>0$, we define the semi-norm:

$$
|u|_{r_{H_{0}(I)}}^{2}:=\left\|_{t}^{r} D_{T}^{\sigma} u\right\|_{L_{2}(I)}^{2},
$$

and norm:

$$
\|u\|_{r_{H_{0}(I)}}:=\left(\|u\|_{L_{2}(I)}^{2}+|u|_{r_{H_{0}^{\sigma}(I)}}^{2}\right)^{\frac{1}{2}},
$$

we then define ${ }^{r} H_{0}^{\sigma}(\Omega)$ as the closure of $C_{0}^{\infty}(\Omega)$ with respect to the norm $\|\cdot\|_{r_{H_{0}^{\sigma}}(\Omega)}$. 
Definition 2.3.

For $\sigma \in \mathbb{R}_{+}, \sigma \neq n+\frac{1}{2}$, we define the semi-norm:

$$
|u|_{c_{H^{\sigma}(I)}}=\left|\left({ }^{R} D_{t}^{\sigma} u,{ }_{t}^{R} D^{\sigma} u\right)_{L^{2}(I)}\right|^{1 / 2}
$$

and norm:

$$
\|u\|_{c_{H^{\sigma}(I)}}=\left(\|u\|_{L^{2}(I)}^{2}+|u|_{c_{H^{\sigma}(I)}}^{2}\right)^{1 / 2} .
$$

we then define ${ }^{c} H^{\sigma}(I)$ as the closure of $C_{0}^{\infty}(I)$ with respect to the norm $\|\cdot\|_{c_{H^{\sigma}(I)}}$.

Lemma 2.1.

[10] For any real $\sigma \in \mathbb{R}_{+}$and let $I=(0, T)$, if $u \in{ }^{l} H^{\alpha}(I)$ and $v \in C_{0}^{\infty}(I)$, then

$$
\left({ }^{R} D_{t}^{\sigma} u(t), v(t)\right)_{L^{2}(I)}=\left(u(t),{ }_{t}^{R} D^{\sigma} v(t)\right)_{L^{2}(I)} .
$$

Lemma 2.2.

$[10,11]$ For $0<\sigma<2, \sigma \neq 1, u \in H_{0}^{\frac{\sigma}{2}}(I)$, we have

$$
{ }^{R} D_{t}^{\sigma} u(t)={ }^{R} D_{t}^{\frac{\sigma}{2}} R D_{t}^{\frac{\sigma}{2}} u(t)
$$

Lemma 2.3.

[10, 11] For $\sigma \in \mathbb{R}_{+}, \sigma \neq n+\frac{1}{2}$, the semi- norms $|\cdot|_{l_{H^{\sigma}(I)}},|\cdot|_{r_{H^{\sigma}(I)}}$ and $|\cdot|_{c_{H^{\sigma}(I)}}$ are equivalent. Then we pose

$$
|\cdot|_{H^{\sigma}(I)} \cong|\cdot|_{r_{H^{\sigma}(I)}} \cong|\cdot|_{c_{H^{\sigma}(I)}} \text {. }
$$

Lemma 2.4.

[10] For any real $\sigma>0$, the space ${ }^{r} H_{0}^{\sigma}(I)$ with respect to the norm (2.20) is complete.

\section{Definition 2.4.}

We denote by $L^{2}\left(0, T, L_{2}(0,1)\right):=L_{2}(Q)$ the space of functions which are square integrable in the Bochner sense, with the scalar product

$$
(u, w)_{L_{2}\left(0, T, L^{2}(0,1)\right)}=\int_{0}^{T}((u, \cdot),(w, \cdot))_{L^{2}(0,1)} d t .
$$

Since the space $L^{2}(0, T)$ is a Hilbert space, it can be shown that $L_{2}\left(0, T, L_{2}(0,1)\right)$ is a Hilbert space as well. Let $C^{\infty}(0, T)$ denote th space of infinitely diffrentiable functions on $(0, T)$ and $C_{0}^{\infty}(0, T)$ denote th space of infinitely diffrentiable functions with compact support in $(0, T)$.

\section{Lemma 2.5.}

Suppose that $\Omega$ is a bounded or unbounded domain in $\mathbb{R}^{n}$. Let $u_{n}(x), u(x)$ be real functions in $L^{p}(\Omega)$; such that $u_{n}$ strongly converges to $u$ in $L^{p}(\Omega)$. Then, if $1 \leq p<\infty, u_{n}$ has a subsequence almost everywhere converging to $u$; if $p=1$, then $u_{n}$ itself almost everywhere converges to $u$. 


\section{Position and solvability of problem}

Consider the following initial boundary value problem for a nonlinear parabolic equation

$$
\begin{cases}{ }_{0}^{C} D_{t}^{\alpha} v-\Delta v=f(x, t) & (x, t) \in \Omega \times(0, T) \\ v(x, t)=0, & (x, t) \in \partial \Omega \times(0, T) \\ v(x .0)=v_{0}(x) . & x \in \Omega\end{cases}
$$

Now, we shall introduce a new function :

$$
u(x, t)=v(x, t)-U(x) \Longrightarrow v(x, t)=u(x, t)+U(x)
$$

where

$$
\varphi(x)=U(x)
$$

So, we get :

$$
\begin{cases}{ }_{0}^{C} D_{t}^{\alpha} u-\Delta u=f(x, t) & (x, t) \in \Omega \times(0, T) \equiv Q . \\ u(x, t)=0, & (x, t) \in \partial \Omega \times(0, T) \\ u(x .0)=0 . & x \in \Omega\end{cases}
$$

Where $\Omega$ is bouded domain in $\mathbb{R}^{n}$ with the smooth boundary $\partial \Omega, f \in L^{2}\left((0, T), L^{2}(\Omega)\right) \equiv L^{2}(Q)$. However , we solve this problem by just following the three major steps described by the Faedo-Galerkin method.

(i) Being the eigenfunctions of the Laplacien operator subject to the Dirichlet boundary conditions

$$
\left\{\begin{array}{c}
-\Delta w_{j}=\lambda_{j} w_{j} \\
w_{j \mid \partial \Omega}=0
\end{array}\right.
$$

We also normalize $w_{j}$ such that $\left\|w_{j}\right\|_{L^{2}(0,1)}=1$. By the elliptic operator theory, $\left\{w_{j}, j \in \mathbb{N}\right\}$ forms base functions in $H_{0}^{1}(\Omega)$, and if $\partial \Omega$ is $C^{\infty}(\bar{\Omega}), j \in \mathbb{N}$. Now we use the Faedo-Galerkin method to find the approximative solution. Let $m$ be a given positive integer and

$$
u_{m}=\sum_{i=1}^{m} g_{i m}(t) w_{i}(x)
$$

Which satisfies the following identities

$$
\left\{\begin{array}{c}
\left(\begin{array}{l}
C \\
0
\end{array} D_{t}^{\alpha} u_{m}, w_{i}\right)_{L^{2}(Q)}-\left(\Delta u_{m}, w_{i}\right)_{L^{2}(Q)}=\left(f, w_{i}\right)_{L^{2}(Q)}, i=1, \ldots, m \\
\left(0, w_{i}\right)_{L^{2}(Q)}=0
\end{array}\right.
$$

where $(.,$.$) denotes the inner product in L^{2}(Q)$. 
Thus,(11) is reducted to the initial value problem for a system of first-order differential equations with respect to $g_{i m}:$

$$
\left\{\begin{array}{cc}
{ }_{0}^{C} D_{t}^{\alpha} g_{i m}+\lambda_{i} g_{i m}=f_{i}(t), & i=1, \ldots, m \\
g_{i m}(0)=0, & i=1, \ldots, m
\end{array}\right.
$$

with $f_{i}=\left(f_{i}, w_{i}\right) \in L^{2}(0, T)$. Thus, there are different methods to solve fractional differential equations analytically or by the usual iteration method used in ODE. One of the most common and widely used methods is the Laplace transform. So, we can conclude that the problem (12) admits a unique local solution $g_{i m}(t)$ such $g_{i m} \in C[0, T]$, and ${ }_{0}^{C} D_{t}^{\alpha} g_{i m} \in L^{2}(0, T)$. (for other methods see references).

(ii) We now try to get the a priori estimates for the approximate solution $u_{m}(x, t)$ obtained in the previous step. Multipliying both sides of equations in (11) by $g_{i m}(t)$, summing with respect to $i$ and integrating over $Q$, we get

$$
\left\|{ }_{0}^{C} D_{t}^{\frac{\alpha}{2}} u_{m}\right\|_{L^{2}(Q)}^{2}+\left\|\nabla u_{m}\right\|_{L^{2}(Q)}^{2}=\left(f, u_{m}\right)_{L^{2}(Q)} .
$$

Using the Cauchy inequality with $\varepsilon$, it comes

$$
\begin{gathered}
\left\|{ }_{0}^{C} D_{t}^{\frac{\alpha}{2}} u_{m}\right\|_{L^{2}(Q)}^{2}+\left\|\nabla u_{m}\right\|_{L^{2}(Q)}^{2} \\
\leq \frac{1}{2 \varepsilon} \int_{0}^{T}\|f\|_{L^{2}(\Omega)}^{2} d \tau+\frac{\varepsilon}{2} \int_{0}^{T}\left\|u_{m}\right\|_{L^{2}(\Omega)}^{2} d \tau
\end{gathered}
$$

By using Poincaré inequality, there exists a constant $c_{\Omega}$, depending only on $\Omega$ we find

$$
\left(\frac{1}{c_{\Omega}}-\frac{\varepsilon}{2}\right)\left\|u_{m}\right\|_{L^{2}(Q)}^{2}+\left\|{ }_{0}^{C} D_{t}^{\alpha} u_{m}\right\|_{L^{2}(Q)}^{2} \leq C_{T} \quad \forall t \in[0, T]
$$

where $C_{T}$ is a positive constant depending only on $\int_{0}^{T}\|f\|^{2} d \tau$ and $T$. It follows from (15) that

$$
\left\|u_{m}(t)\right\|_{L^{2}(Q)}^{2}=\sum_{i=1}^{m} g_{i m}^{2}(t) \leq C_{T} \quad \forall t \in[0, T]
$$

This implies that the solution to the initial value problem for the system of ODE (12) can be extended to $[0, T]$ and on $[0, T]$, we have the following uniform a priori estimates

$$
\begin{array}{lc}
u_{m} & \text { uniformly bounded in } L^{2}\left([0, T], H_{0}^{1}(\Omega)\right), \\
{ }_{0}^{C} D_{t}^{\alpha} u_{m} & \text { uniformly bounded in } L^{2}\left([0, T], L^{2}(\Omega)\right) .
\end{array}
$$

(iii) Since any bounded set in a reflexive Banach space is weakly compact, i.e., any sequence in a bounded set has a weakly converging subsequence. Then, there is a subsequence of $u_{m}$, still denoted by $u_{m}$ such that

$$
\left\{\begin{array}{l}
u_{m} \longrightarrow u \quad \text { weakly in } L^{2}\left([0, T], H_{0}^{1}(\Omega)\right) \\
{ }_{0}^{C} D_{t}^{\alpha} u_{m} \longrightarrow{ }_{0}^{C} D_{t}^{\alpha} u \quad \text { weakly in } L^{2}(Q)
\end{array} .\right.
$$


We know that according to Rellich-Kondrachoff's theorem (see Lions-Magenes [14]; the embedding of $H_{1}(Q)$ in $L^{2}(Q)$ is compact. So there is a subsequence of $u_{m}$, still denoted by $u_{m}$ such that $u_{m}$ strong converges to $u$ in $L^{2}\left([0, T], H_{0}^{1}(\Omega)\right)$.

By Lemma 5 there is a subsequence of $u_{m}$, still denoted by $u_{m}$ almost everywhere converges to $u$ in $Q$.

Passing to the limit in (11), since each term on the left-hand side of $(11)$ is weakly convergent in $L^{2}([0, T])$, we obtain that the following holds in $L^{2}([0, T])$ :

$$
\left({ }_{0}^{C} D_{t}^{\alpha} u, w_{i}\right)-\left(\Delta u, w_{i}\right)=\left(f, w_{i}\right), \quad \forall i=1,2 \ldots
$$

Since $\left\{w_{i}, i \in \mathbb{N}\right\}$ is a base in $L^{2}(\Omega)$, we infer from (17) that the following holds in $L^{2}\left([0, T], L^{2}(\Omega)\right)$ :

$$
{ }_{0}^{C} D_{t}^{\alpha} u-\Delta u=f
$$

Since all ${ }_{0}^{C} D_{t}^{\alpha} u, \Delta u$, and $f$ belong to $L^{2}\left([0, T], L^{2}(\Omega)\right)$, so $(18)$ also belongs to $L^{2}\left([0, T], L^{2}(\Omega)\right)$. Thus, we have the following result.

Theorem 3.1.

Suppose that $f \in L^{2}\left([0, T], L^{2}(\Omega)\right)$. The problem (8) admits a solution u such that

$$
u \in L^{2}\left([0, T],^{l} H^{\frac{\alpha}{2}}(\Omega)\right) \cap L^{2}\left([0, T], H_{0}^{1}(\Omega)\right)
$$

Proof. Existence of a solution has been proved through three steps described previously.

\section{Estimation a priori "Uniqueness of the solutions"}

The method used here is one of the most efficient functional analysis methods and important techniques for solving partial differential equations, which has been successfully used in investigating the existence, uniqueness, and continuous dependence of the solutions of PDE's, the so-called a priori estimate method or the energyinequality method. This method is essentially based on the construction of multiplicators for each specific given problem, which provides the a priori estimate from which it is possible to establish the solvability of the posed problem as a solution of the operator equation

$$
L u=\text { Fourier }
$$

where $L:=($ Laplace, $\ell)$, with domain of definition $B$ consisting of functions $u \in L^{2}(Q)$, such that $u,{ }^{C} D_{t}^{\alpha} u, \frac{\partial u}{\partial x} \in$ $L^{2}(Q)$.

The operator $L$ is considered from $B$ to $F$, where $B$ is the Banach space consisting of all functions $v(x, t)$ having a finite norm

$$
\|u\|_{B}^{2}=\left\|{ }_{0}^{C} D_{t}^{\frac{\alpha}{2}} u\right\|_{L^{2}(Q)}^{2}+\|u\|_{L^{2}\left(Q_{T}\right)}^{2} .
$$


with $F$ is the Hilbert space consisting of all elementsFourier $\equiv(f, \ell)$, where $\ell u=0$; for which the norm in $L^{2}(Q)$ is finite.

\section{Theorem 4.1.}

For any function $u \in B$, we have the inequality

$$
\|u\|_{B} \leq k\|L u\|_{L^{2}(Q)}
$$

where $k$ is a positive constant independent of $u$.

Proof. Multiplying the equation (3.1) by the following function :

$$
M u=u(x, t),
$$

and integrating over $Q$, we get :

$$
\begin{aligned}
& \int_{Q} \text { Laplaceu } \cdot \text { Mudxdt } \\
= & \int_{Q}{ }_{0}^{C} D_{t}^{\alpha} u(x, t) \cdot u(x, t) d x d t-\int_{Q}(\Delta u) \cdot u(x, t) d x d t \\
= & \int_{Q} f(x, t) \cdot u(x, t) d x d t .
\end{aligned}
$$

As $u(x, 0)=0$, we have ${ }_{0}^{C} D_{t}^{\alpha} u(x, t)={ }_{0}^{R} D_{t}^{\alpha} u(x, t)$, so by applying Lemmas 1,2 and 3 , we obtain

$$
\begin{aligned}
& \int_{Q}{ }_{0}^{C} D_{t}^{\alpha} u(x, t) \cdot u(x, t) d x d t \\
& =\left({ }_{0}^{C} D_{t}^{\alpha} u(x, t), u(x, t)\right)_{L^{2}(Q)} \\
& =\left({ }_{0}^{C} D_{t}^{\frac{\alpha}{2}}{ }_{0}^{C} D_{t}^{\frac{\alpha}{2}} u(x, t), u(x, t)\right)_{L^{2}(Q) \quad \text { (According to Lemma 2) }} \\
& =\left({ }_{0}^{C} D_{t}^{\frac{\alpha}{2}} u(x, t){ }_{t}^{C} D_{T}^{\frac{\alpha}{2}} u(x, t)\right)_{L^{2}(Q) \quad \text { (According to Lemma 1) }} \\
& =|u|_{c H^{\alpha}(Q)}^{2} \cong|u|_{c H^{\alpha}(Q)}^{2}=\left\|{ }_{0}^{C} D_{t}^{\frac{\alpha}{2}} u\right\|_{L^{2}(Q)}^{2}, \quad \text { (According to Lemma 3) }
\end{aligned}
$$

and by integration by parts over $Q$; we get :

$$
-\int_{Q} \int_{Q}(\Delta u) \cdot u(x, t) d x d t=\int_{Q}(\nabla u)^{2} d x d t .
$$

So, we obtain :

$$
\begin{aligned}
& \int_{Q}\left({ }_{0}^{C} D_{t}^{\alpha} u-\Delta u\right) \cdot M v d x d t \\
\cong & \left\|{ }_{0}^{C} D_{t}^{\frac{\alpha}{2}} u\right\|_{L^{2}(Q)}^{2}+\|\nabla u\|_{L^{2}(Q)}^{2} \\
= & \int_{Q} f(x, t) \cdot u(x, t) d x d t .
\end{aligned}
$$


By using the Cauchy inequality with $\varepsilon$, it comes

$$
\int_{Q} f(x, t) \cdot u(x, t) d x d t \leq \frac{1}{2 \varepsilon} \int_{Q}|f(x, t)|^{2} d x d t+\frac{\varepsilon}{2} \int_{Q}|u(x, t)|^{2} d x d t
$$

By using Poincaré inequality, there exists a constant $c_{\Omega}$, depending only on $\Omega$ we find

$$
\begin{aligned}
& \left\|\begin{array}{l}
C \\
0
\end{array} D_{t}^{\frac{\alpha}{2}} u\right\|_{L^{2}(Q)}^{2}+\left(\frac{1}{c_{\Omega}}-\frac{\varepsilon}{2}\right)\|u\|_{L^{2}(Q)}^{2} \\
\leq & \frac{1}{2 \varepsilon}\|f\|_{L^{2}(Q)}^{2} .
\end{aligned}
$$

Finally, it follows that

$$
\left\|{ }_{0}^{C} D_{t}^{\frac{\alpha}{2}} u\right\|_{L^{2}(Q)}^{2}+\|u\|_{L^{2}(Q)}^{2} \leq C\|f\|_{L^{2}(Q)}^{2},
$$

with

$$
C=\frac{1}{2 \varepsilon}\left(\frac{1}{\min \left\{1 ;\left(\frac{1}{c_{\Omega}}-\frac{\varepsilon}{2}\right)\right\}}\right)
$$

Therefore, we obtain that

$$
\|v\|_{B} \leq k\|L v\|_{F}, \quad \text { where } k=\sqrt{C} .
$$

Hence the uniqueness of the solution.

Remark 4.1.

This inequality

$$
\|v\|_{B} \leq k\|L v\|_{F}
$$

is gives the uniqueness of the solution, indeed:

Let $v_{1}$ and $v_{2}$ two solutions, so

$$
\left\{\begin{array}{l}
L v_{1}=\text { Fourier } \\
L v_{2}=\text { Fourier }
\end{array} \Longrightarrow L\left(v_{1}-v_{2}\right)=0\right.
$$

then

$$
\left\|v_{1}-v_{2}\right\|_{B} \leq k\|0\|_{F} \Longrightarrow\left\|v_{1}-v_{2}\right\|_{B} \leq 0 \Longrightarrow v_{1}-v_{2}=0
$$

which gives the uniqueness of the solution.

\section{Statements and Declarations}

All authors have no conflict of interest to report. 


\section{References}

[1] A. A. Kilbas, H. M. Srivastava, J. J. Trujillo, Theory and Applications of Fractional Differential Equations, Elsevier, Amsterdam, 2006.

[2] J. H. He, Nonlinear oscillation with fractional derivative and its applications. In: International Conference on Vibrating Engineering'98, Dalian, China (1998), 288-291.

[3] J. H. He, Some applications of nonlinear fractional differential equations and their approximations, Bull. Sci. Technol. 15 (1999), 86-90.

[4] J. H. He, Approximate analytical solution for seepage flow with fractional derivatives in porous media, Comput. Methods Appl. Mech. Eng. 167 (1998), 57-68.

[5] K. S. Miller, B. Ross, An Introduction to the Fractional Calculus and Differential Equations, J. Wiley, New York, 1993.

[6] S. G. Samko, A. A. Kilbas, O. I. Marichev, Fractional Integrals and Derivatives: Theory and Applications, Gordon and Breach, Yverdon, 1993.

[7] R. P. Agarwal, M. Benchohra, S. Hamani, Boundary value problems for fractional differential equations, Adv. Stud. Contemp. Math. 16 (2008), 181-196.

[8] T-E. Oussaeif, A. Bouziani, Existence and uniqueness of solutions to parabolic fractional differential equations with integral conditions, Electron. J. Differ. Equ. 2014 (2014), 1-10.

[9] O. Taki-Eddine, B. Abdelfatah, A priori estimates for weak solution for a time-fractional nonlinear reactiondiffusion equations with an integral condition, Chaos, Solitons \& Fractals 103 (2017), 79-89.

[10] X. J. Li, C. J. Xu, Existence and uniqueness of the weak solution of the space-time fractional diffusion equation and a spectral method approximation, Communications in Computational Physics 8 (2010), 1016-1051.

[11] X. J. Li, C. J. Xu, A space-time spectral method for the time fractional diffusion equation, SIAM Journal on Numerical Analysis 47 (2009), 2108-2131.

[12] N. J. Ford, J. Xiao, Y. A. Yan, A finite element method for time fractional partial differential equations, Fractional Calculus Appl. Anal. 14 (2011), 454-474.

[13] O. Zigen, Existence and uniqueness of the solutions for a class of nonlinear fractional order partial differential equations with delay, Comput. Math. Appl. 61 (2011), 860-870.

[14] J. L. Lions, E. Magenes, Problémes aux limites non homogènes et applications, vol. 1 and 2, Paris, Dunod, 1968.

[15] O. Taki-Eddine, B. Abdelfatah, A priori estimates for weak solution for a time-fractional nonlinear reactiondiffusion equations with an integral condition, Chaos, Solitons \& Fractals 103 (2017), 79-89.

[16] T-E. Oussaeif, A. Bouziani, Mixed problem with an integral two-space-variables condition for a class of hyperbolic equations, International Journal of Analysis 2013 (2013).

[17] T-E. Oussaeif, A. Bouziani, Mixed problem with an integral two-space-variables condition for a parabolic 
equation, International Journal of Evolution Equations 9 (2013), 181-198.

[18] B. Abdelfatah, O. Taki-Eddine, B. A. Leila, A mixed problem with an integral two-space-variables condition for parabolic equation with the bessel operator, Journal of Mathematics 2013 (2013), Article ID 457631.

[19] A. Benaoua, O. Taki-Eddine, I. Rezzoug, Unique solvability of a Dirichlet problem for a fractional parabolic equation using energy-inequality method, Methods Funct. Anal. Topology 26 (2020), 216-226.

[20] G. Bahia, A. Ouannas, I. M. Batiha, Z. Odibat, The optimal homotopy analysis method applied on nonlinear time-fractional hyperbolic partial differential equations, Numerical Methods for Partial Differential Equations 37 (2021), 2008-2022.

[21] M. T. Shatnawi, A. Ouannas, G. Bahia, I. M. Batiha, G. Grassi, The optimal homotopy asymptotic method for solving two strongly fractional-order nonlinear benchmark oscillatory problems, Mathematics 9 (2021), Article ID 2218. 